\title{
Spatial landslide hazard assessment along the Jelapang Corridor of the North-South Expressway in Malaysia using high resolution airborne LiDAR data
}

\begin{abstract}
Mapping landslide-prone regions are crucial in natural hazard management and urban development activities in hilly and tropical regions. This research aimed to delineate a spatial prediction of landslide hazard areas along the Jelapang Corridor of the North-South Expressway in Malaysia by using two statistical models, namely, logistic regression (LR) and evidential belief function (EBF). Landslides result in high economic and social loses in Malaysia, particularly to highway concessionaries such as PLUS Expressways Berhad. LR and EBF determine the correlation between conditioning factors and landslide occurrence. EBF can also be applied in bivariate statistical analysis. Thus, EBF can be used to assess the effect of each class of conditioning factors on landslide occurrence. A landslide inventory map with 26 landslide sites was recorded using field measurements. Subsequently, the landslide inventory was randomly divided into two data sets. Approximately $70 \%$ of the data were used for training the models, and $30 \%$ were used for validating the results. Eight landslide conditioning factors were prepared for landslide susceptibility analysis: altitude, slope, aspect, curvature, stream power index, topographic wetness index, terrain roughness index, and distance from river. The landslide probability index was derived from both methods and subsequently classified into five susceptible classes by using the quantile method. The resultant landslide susceptibility maps were evaluated using the area under the curve technique. Results revealed the proficiency of the LR method in landslide susceptibility mapping. The achieved success and prediction rates for LR were 90 and $88 \%$, respectively. However, EBF was not successful in providing reasonable accurate results. The acquired success and prediction rates for EBF were 53 and $50 \%$, respectively. Hence, the LR technique can be utilized in landslide hazard studies for land use management and planning.
\end{abstract}

Keyword: Landslide; Hazard; LiDAR; EBF; LR; GIS; Malaysia 